\title{
Die Entwicklung des prosozialen Verhaltens in den ersten zwei Lebensjahren
}

\section{Silvana Kappeler und Heidi Simoni}

Die Entwicklung des prosozialen Verhaltens wurde längsschnittlich an 28 Kinder vom 9. Lebensmonat bis zum 25. Monat mittels teilnehmender Beobachtung im natürlichen Setting untersucht. Prosoziales Verhalten wurde definiert als Handlungen, die das Wohlbefinden einer anderen Person bzw. einer Gruppe erhöhen oder wiederherstellen oder eine andere Person bzw. eine Gruppe begünstigen sollen. Es wurden sechs Formen des prosozialen Verhaltens untersucht: Assistieren, Trösten, Zuneigung zeigen, Objekte anbieten, Wiedergutmachung sowie prosoziales Verhalten gegenüber Objekten.

Erste Formen, das Anbieten von Objekten, zeigten einzelne Kinder bereits mit 8 Monaten, bis zum Alter von 16 Monaten konnten sämtliche der untersuchten Formen beobachtet werden. Einen grossen Teil des prosozialen Verhaltens führten die Kinder spontan aus, ohne dazu aufgefordert worden zu sein. Die einzelnen Formen unterschieden sich jedoch beträchtlich hinsichtlich Erstmanifestation, Häufigkeit und zeitlichem Verlauf. Dies deutet darauf hin, dass den verschiedenen prosozialen Verhaltensweisen unterschiedliche sozio-kognitive Fähigkeiten zugrunde liegen. Es konnten Geschlechtsunterschiede nachgewiesen werden: Mädchen handelten häufiger prosozial als Jungen.

Die Fähigkeit, sich anderen gegenüber in einer positiven Weise zu verhalten, das heisst, Mitgefühl zu zeigen, zu trösten, zu helfen oder einander Zuneigung zu bekunden, kann als zentraler Aspekt der sozialen Kompetenz gelten und wird unter dem Begriff prosoziales Verhalten zusammengefasst.

Lange wurde angenommen, dass sich die Fähigkeit, prosozial zu handeln ausserhalb des Repertoires von Kleinkindern befindet. In der klassischen psychoanalytischen Theorie (Freud, 2009), in der kognitiven Entwicklungstheorie von Piaget (1981) und der Theorie zur moralischen Entwicklung nach Kohlberg (1976) wurden Kinder als amoralisch, egozentrisch und sozial unreif dargestellt. Erst mit der Überwindung des Egozentrismus ${ }^{1}$ resp. der Bildung des Über-Ichs als Folge der Lösung der Ödipus-Situation mit ca. 5 - 6 Jahren hätten die Kinder als notwendig erachteten Voraussetzungen erworben, um prosozial handeln zu können. 
Dem widersprechen jedoch neuere theoretische Entwicklungen und Forschungsarbeiten (z.B. Keller, 2005; Vaish, Carpenter \& Tomasello, 2009; Warneken \& Tomasello, 2007) sowie Alltagsbeobachtungen. Dass bereits Kinder unter zwei Jahren prosozial reagieren, zeigt die folgende Episode eines Vaters mit seinem 14 Monate alten Sohn:

Als wir an dem Nachmittag nach Hause kamen, rutschte ich aus und fiel auf die Nase. Es tat sehr weh, und ich setzte mich in den Schaukelstuhl in J.'s Zimmer und rieb mir die Nase. J. war sehr mitfüblend. Er tat das für mich, was ich sonst für ihn tue, wenn er sich verletzt. Er umarmte und tätschelte mich und bot mir sogar seine Decke an, die er nimmt, wenn er sich wehgetan hat oder müde ist. Er schien sehr besorgt meinetwegen. (Wolf, 1982, zitiert nach Harris, 1992, S. 36)

Diese Begebenheit zeigt eindrücklich, dass offensichtlich schon Kleinkinder fähig sind, sich in die Lage des anderen zu versetzen und prosozial zu handeln, wenn die Situation innerhalb ihres Erfahrungshorizontes liegt.

Hoffman $(1975,1982)$ versuchte mit seiner Empathietheorie, einseitig auf kognitive Faktoren fokussierten Ansätzen entgegenzuwirken. Er konzentriert sich auf das Helfen in emotionalen Notlagen und die Wiedergutmachung, wobei er für die Empathie - als stellvertretende emotional-mitfühlende Reaktion gegenüber einer anderen Person - eine zentrale Rolle bei der Auslösung annimmt. Im reflexiven Weinen von Neugeborenen als Reaktion auf das Weinen anderer Kinder sieht Hoffman eine biologische Prädisposition für die Empathie, die er als primär affektive Reaktion versteht. Trotzdem kommt kognitiven Faktoren in seiner Theorie eine zentrale Stellung zu, da der Stand der sozio-kognitiven Entwicklung die Stufen der Empathieentwicklung bestimmt. Erst wenn die Kinder im zweiten Lebensjahr in der Lage sind, sich kognitiv von anderen zu unterscheiden und somit eine Vorstellung von anderen als eigenständige Lebewesen entwickelt haben, transformiert sich ihre emotionale Reaktion auf das Leid anderer von einer auf des Selbst fokussierten Stressreaktion in empathische Sorge und Mitgefühl um die andere Person. Hoffman nimmt an, dass erste prosoziale Initiativen auf das Leid anderer in dieser Phase auftreten. Neben der Empathie schreibt Hoffman auch der Schuld motivationales Potential zu, wobei beide ähnliche Entwicklungslinien aufweisen. Während jedoch die Empathie als Reaktion auf das Leid einer anderen Person auftritt, unabhängig davon, ob das Kind das Leid der anderen Person lediglich beobachtet oder es verursacht, resultiert die Schuld aus einer empathischen Reaktion auf das Leid einer anderen Person und der Erkenntnis, dieses verursacht zu haben.

Bischof-Köhler (1989) baut ihr Modell zur Entwicklung empathischer Reaktionen auf Hoffmans Theorie auf und differenziert diese weiter. Sie weist insbesondere auf die Notwendigkeit hin, Empathie und Gefühlsansteckung voneinander abzugrenzen: Bei der Gefühlsansteckung, die Hoffmans reflexivem Weinen entspricht, ergreift die Stimmung des anderen vom Beobachtenden Be- 
sitz und wird zu dessen eigenem Gefühl. Von Empathie hingegen spricht Bischof-Köhler (1989) dann, wenn der Beobachter unmittelbar der Gefühlslage eines anderen teilhaftig wird, das übertragene Gefühl aber als der anderen Person zugehörig erkennt. Voraussetzung hierfür ist die Ausbildung eines Selbstkonzepts, das eine Ich-Andere-Unterscheidung ermöglicht. Dadurch kann ein Kind sich seiner selbst als eines vom anderen abgegrenzten Erlebnisträgers bewusst werden. Bischof-Köhler spricht in diesem Zusammenhang auch von der Fähigkeit zur Selbstobjektivierung. Auf dem Mechanismus der Gefühlsansteckung aufbauend kann Empathie resultieren, sobald zwischen dem 18. und 24. Lebensmonat ein wie oben beschriebenes Selbstkonzept ausgebildet ist. Damit ist die kognitive Voraussetzung dafür gegeben, ein mitempfundenes Gefühl als Gefühl des anderen zu erkennen und daraus auf dessen emotionale Verfassung zu schliessen.

Karniol (1982) dagegen stützt sich bei der Erklärung prosozialen Verhaltens auf Informationsverarbeitungsmodelle und nicht auf Faktoren wie die Ich-Andere-Unterscheidung und die Empathie. Wenn Wissen über Hilfesituationen und Möglichkeiten der Hilfeleistung gespeichert ist, wird die Situation verstanden und ein Handlungsplan liegt bereit. Allerdings helfen kleine Kinder nur dann, wenn der potentielle Empfänger ihnen vertraut ist oder sie wollen, dass er sie mag.

Kochanska (1984) wiederum geht davon aus, dass die Persönlichkeit aus verschiedenen Subsystemen besteht, die in komplexer Weise miteinander zusammenhängen. Obwohl jedes dieser Subsysteme prosoziales Verhalten beeinflussen kann, sind die zugrunde liegenden Mechanismen unterschiedlich. Prosoziales Verhalten, das dem emotionalen System entspringt, beruht auf Empathie, wie sie Hoffman beschreibt. Wenn das Kind bevorzugt Personen hilft, die es als ähnlich wahrnimmt, stammt dieses Verhalten aus dem Subsystem der Selbststruktur. Dieses ist ebenfalls die Quelle für prosoziales Verhalten, welches der Erhöhung des eigenen Wohlbefindens oder der Erlangung einer externalen Belohnung dient. Der autonome Mechanismus führt zu prosozialem Verhalten, wenn die Repräsentationen von anderen Menschen einen positiven Wert besitzen. Die kognitiven Voraussetzungen, dafür sind bei Kochanska identisch mit der von Hoffman beschriebenen Entwicklung.

Trotz unterschiedlicher Schwerpunkte ist den referierten Theorien gemeinsam, dass sie davon ausgehen, dass bereits Kinder unter zwei Jahren fähig sind, prosozial zu handeln.

Diese Annahme wird durch empirische Ergebnisse zunehmend und immer differenzierter gestützt.

Stanjek (1978) führte eine Serie von Studien zum Überreichen von Objekten durch. In der ersten Studie wurden 20 Kinder im Alter von 19 - 37 Monaten in der Kindertagesstätte während des Freispiels beobachtet. Dabei wurden Verhaltensweisen erfasst, die zu Kontakten der Kinder untereinander und zu Erwachsenen führten. Jedes Kind wurde insgesamt fünf Mal 10 Minuten lang beobach- 
tet. Es zeigte sich, dass das Überreichen von Objekten zur Herstellung eines Kontaktes sehr häufig vorkam, allerdings mit einem deutlichen Alterstrend: Bei älteren Kindern wurde es zunehmend durch andere Formen der Kontaktaufnahmen wie Ansprechen ersetzt. In der zweiten Studie wurde das Kontaktverhalten von 24 Kindern im Alter von 17 - 37 Monaten gegenüber fremden Personen, die die Kindertagesstätten besuchten, untersucht. Auch in dieser Untersuchung waren das Überreichen und das Zeigen von einfachen Holzstücken, Legosteinen oder anderem Spielmaterial ein häufiges Verhalten bei der Kontaktaufnahme. Die dritte Untersuchung fand im Wartezimmer eines Kinderkrankenhauses statt. Auch hier war das Übergeben von Objekten ein häufiges Element, mit dem die Kinder im Alter zwischen 1 - 7 Jahren Interaktionen begannen und zwar sowohl mit den Eltern wie auch mit fremden Kindern oder Erwachsenen.

Rheingold (1982) untersuchte in ihrer Studie das Assistieren, also Verhaltensweisen, die zur Komplettierung einer Aufgabe beitragen. An der Studie nahmen insgesamt 80 Kinder im Alter von 18, 24 und 30 Monaten mit ihren Eltern teil. In der Untersuchung verrichteten die Mütter, Väter oder eine dem Kind unbekannte Person im Labor alltägliche Haushaltsarbeiten. Beinahe alle Kinder, auch die jüngsten, zeigten assistierendes Verhalten: bei den 18 Monate alten Kindern 13 von 20, bei den 24 Monate alten Kindern alle der ersten und 18 von 20 der zweiten Gruppe sowie bei den 30 Monate alten Kindern alle 20. Der Prozentsatz des Helfens war bei den älteren Kindern etwas höher: Die jüngsten Kinder halfen bei 63\%, die mittleren bei 78\% und die ältesten bei 89\% der Aufgaben. Geschlechtsunterschiede wurden keine gefunden.

Zahn-Waxler und Radke-Yarrow (1982) untersuchten an 24 Kindern, die zu Beginn der Studie 10, 15 und 20 Monate alt waren, die Entwicklung des prosozialen Verhaltens während neun Monaten. Die Reaktionen der Kinder auf Situationen, in welchen andere Personen negative Emotionen zeigten, wurden von ihren Müttern festgehalten. In der jüngsten Altersgruppe war das Weinen als Reaktion auf Leid anderer vorherrschend. Die erste prosoziale Intervention ereignete sich kurz nach dem ersten Lebensjahr in Form von körperlichem Kontakt wie umarmen und tätscheln. Während des zweiten Lebensjahrs wurde zusätzlich Verhalten wie direkte Hilfe, Teilen, indirekte Hilfe (z. B. die Mutter holen), Hilfevorschläge machen und Mitgefühl ausdrücken bedeutsam. Auf das Selbst orientierte Verhaltensweisen wie Weinen oder Hilfe bei der Mutter suchen wurden graduell durch direkte Kontakte mit dem Opfer ersetzt.

Vaish et al. (2009) untersuchten Reaktionen von Mitgefühl und prosoziales Verhalten an 64 Kindern im Alter von eineinhalb und zwei Jahren. In der ersten Phase (Mitgefühl-Experiment) beobachteten die Kinder, wie eine Versuchsleiterin einer zweiten einen Gegenstand entwendete oder zerstörte (schädigende Variante) oder sich neutral verhielt (neutrale Variante). Die Rezipientinnen des Verhaltens zeigten in beiden Varianten keine besonderen emotionalen Reaktionen. In einer zweiten Phase der Studie (Experiment zum prosozialen Verhalten) übergab eine Versuchsleiterin der anderen einen mit Helium gefüllten Ballon, 
dem Kind zwei Ballone. Nach einer Weile liess die Versuchsleiterin den Ballon «aus Versehen» los, so dass er an die Decke stieg. Die Versuchsleiterin versuchte vergeblich, an den Ballon zu gelangen. Das Verhalten des Kindes wurde während maximal zweier Minuten beobachtet. Reagierte das Kind nicht, stieg die Versuchsleiterin auf einen Stuhl und holte sich den Ballon wieder. Kinder, die in der ersten Phase die schädigende Variante erlebt hatten, halfen der Versuchsleiterin in der zweiten Phase signifikant häufiger als die Kinder der neutralen Variante. Ausserdem korrelierte das Ausmass von Besorgnis, das die Kinder im MitgefühlExperiment gezeigt hatten, positiv mit ihrem prosozialen Verhalten. Dagegen konnten keine Alters- oder Geschlechtsunterschiede festgestellt werden.

Die referierten Studien zeigen, dass bereits Kinder unter zwei Jahren in der Lage sind, verschiedene Formen von prosozialem Verhalten zu zeigen. Trotz des verstärkten Interesses gibt es jedoch bis heute nur wenig Forschung zur Entwikklung des prosozialen Verhaltens im Kleinkindalter. Es mangelt insbesondere an Längsschnittstudien im natürlichen Umfeld des Kindes, die mehrere prosoziale Formen untersuchen. Um den Entwicklungsverlauf zuverlässig zu dokumentieren, ist ein längsschnittlich angelegtes Design jedoch unerlässlich. Beobachtungen im natürlichen Umfeld des Kindes sind aufschlussreich, weil das prosoziale Verhalten von Kleinkindern stark von Merkmalen der aktuellen Situation und des potentiellen Empfängers abhängig ist. Von Interesse ist ferner, ob verschiedene Formen von prosozialem Verhalten ähnliche Fähigkeiten voraussetzen und deshalb denselben Entwicklungslinien folgen oder Teile eines heterogenen Konstruktes sind, die auf unterschiedlichen sozio-kognitiven und emotionalen Fähigkeiten basieren und mit je verschiedenen Merkmalen des Kindes zusammenhängen.

\section{Forschungs fragen}

Mit der hier vorgestellten Untersuchung sollte die frühe Entwicklung des prosozialen Verhaltens längsschnittlich im natürlichen Umfeld des Kindes untersucht werden. Konkret standen folgende Fragen im Mittelpunkt:

- Wie häufig zeigen Kinder prosoziales Verhalten?

- In welchem Alter zeigen Kinder erstmals prosoziales Verhalten?

- Welcher Verlauf lässt sich bis 2 Jahre beobachten?

- Zeigen sich Geschlechtsunterschiede hinsichtlich des prosozialen Verhaltens?

- Wie hängen die verschiedenen Formen von prosozialem Verhalten miteinander zusammen?

Prosoziale Verhaltensweisen wurden in Anlehnung an Eisenberg (1986) definiert als Handlungen, die das Wohlbefinden einer anderen Person bzw. einer Gruppe erhöhen oder wiederherstellen oder eine andere Person bzw. eine Gruppe begünstigen sollen. 
Damit ist eine Voraussetzung verbunden: Es soll beim Akteur die Handlungsintention vorhanden sein, den Empfänger vom Verhalten profitieren zu lassen. Prosoziales Verhalten in diesem weiten Sinne umfasst Aktionen, die aus egoistischen oder aus selbstlosen Motiven ausgeführt werden. So kann ein Kind ein weinendes Kind aus Mitgefühl trösten, aber auch, weil es sich davon eine Belohnung, z. B. ein Lob oder Aufmerksamkeit, verspricht.

Altruistische Motive sind also möglich aber keine Bedingung der Definition. Altruistisches Verhalten bezieht sich - so Eisenberg und Mussen (1989) - auf einen spezifischen Typus des prosozialen Verhaltens, nämlich auf Handlungen, die intrinsisch motiviert sind. Das heisst, altruistisches Verhalten ist motiviert durch Besorgnis und Mitgefühl, persönliche Überzeugungen und Werte sowie SelbstBekräftigung, nicht jedoch durch die Erwartung einer externalen Belohnung oder eines persönlichen Nutzens.

Im Unterschied zu Eisenberg (1986), die prosoziales Verhalten zusätzlich als freiwillig definiert, wurde die Voraussetzung der Freiwilligkeit nicht in die Definition aufgenommen. Dies deshalb, weil prosoziales Verhalten, das als Reaktion auf eine Aufforderung oder Bitte hin ausgeführt wird, ebenfalls erfasst werden sollte.

In der vorliegenden Studie wurde als Grundlage eine weit gefasste Definition verwendet. Zum einen ist der Rekurs auf die Motive der Handlung mit erheblichen Schwierigkeiten verbunden. Was eine Person zum Helfen bewegt, ist dem Untersucher oft nur schwer oder gar nicht zugänglich. Zudem wirken häufig mehrere Motive nebeneinander, so dass es ausserordentlich schwierig ist, die Motivation des Helfers an einem einzigen Motiv festzumachen. Die in der vorliegenden Untersuchung beobachtete Altersgruppe kann zudem noch keine verbalen Angaben über ihre Motive machen. Es erschien deshalb weder sinnvoll noch möglich, zwischen altruistisch motivierten prosozialen Aktionen und solchen, die aus weniger «noblen» Gründen ausgeführt werden, zu unterscheiden. Eine weiter gefasste Definition erleichtert es zum andern Vorläufer prosozialer Verhaltensweisen aufzuspüren und damit zum Verständnis von Entwicklungsprozessen möglicherweise einen Beitrag zu leisten. Dieses Interesse liegt auch der Auswahl der sechs Verhaltensweisen, die in der Untersuchung unterschieden wurden, zugrunde. Es sind dies das Anbieten von Objekten, das Zeigen von $\mathrm{Zu}-$ neigung, Assistieren, Trösten, Wiedergutmachung sowie prosoziales Verhalten gegenüber Objekten (z. B. gegenüber einer Puppe).

\section{Me thode}

Die Studie «Die Entwicklung des prosozialen Verhaltens in den ersten zwei Lebensjahren» basiert auf derselben Stichprobe und Datenerhebung wie die Studie «Erwerb sozialer Kompetenz von Kleinkindern im Kontakt mit Gleichaltrigen und mit Erwachsenen» ${ }^{2}$ des Marie Meierhofer-Instituts für das Kind (vgl. dazu: 
Simoni, Herren, Kappeler \& Licht, 2008). Deshalb werden im Folgenden zuerst die Datengrundlage und die Basisanalysen vorgestellt und danach das spezifische methodische Vorgehen.

\section{St ichprobe}

Es wurden $28(17 \mathrm{w}, 11 \mathrm{~m})$ normal entwickelte Kinder, die regelmässig familienergänzende Betreuungseinrichtungen besuchten, in die Untersuchung aufgenommen. Die Mehrheit der Kinder stammte aus Familien der sozialen Mittelschicht mit einem eher hohen Ausbildungsniveau (76\% der Eltern hatten eine akademische oder eine äquivalente Ausbildung). Knapp die Hälfte aller Kinder (46\%) hatte mindestens ein Geschwister. In den meisten Familien (89\%) waren beide Eltern zumindest teilzeitlich berufstätig. 11\% der Kinder lebten bei allein erziehenden Müttern. Die Rekrutierung der Stichprobe erfolgte in Kooperation mit Kinderkrippen im Grossraum Zürich: Jedes 8 Monate alte Kind, dessen Eltern ihr Einverständnis dazu gegeben hatten, wurde als Zielkind in die Stichprobe aufgenommen. Die andern Kinder, die dieselbe Krippengruppe wie das Zielkind besuchten, wurden bei vorliegender Einwilligung ihrer Eltern als Kontaktkinder einbezogen. Ihr Alter lag zwischen sechs Monaten und sechs Jahren.

\section{Datenerhebung}

Die Hauptmethode der Studie bestand in der längsschnittlichen Beobachtung der Zielkinder im natürlichen Setting. Jedes Zielkind wurde in seiner Kinderkrippe ab dem neunten Lebensmonat bis zum Alter von 24 Monaten neun Mal im Abstand von zwei Monaten während 30 Minuten gefilmt. Die Videoaufnahmen fanden in Freispielphasen in der Krippe und meistens am Vormittag statt. Freispielsituationen sind dadurch gekennzeichnet, dass die Kinder selbst entscheiden können, welchen Aktivitäten sie nachgehen und die Situation nicht von der Erzieherin strukturiert oder gestaltet wird. Dieser Kontext gestaltet die Situationen zwischen den einzelnen Zielkindern vergleichbar und ermöglicht ein hohes Mass an selbstregulierter Tätigkeit. Andererseits wird verhindert, dass das Verhalten der Zielkinder zu sehr von den Erzieherinnen beeinflusst wird. Bei den Videoaufnahmen handelte es sich um eine teilnehmende Beobachtung, das heisst, die wissenschaftliche Mitarbeiterin war in den Räumlichkeiten anwesend und filmte die Aktivitäten des Zielkindes mittels einer Handkamera. Eine nicht teilnehmende Beobachtung war aufgrund der Ausstattung der Kindertagesstätten nicht möglich. Es zeigte sich jedoch, dass sich die Kinder sehr schnell an die Filmerin und die Videokamera gewöhnten und diese schon nach kurzer Zeit nicht mehr beachteten. Um diese subjektiven Eindrücke abzusichern, wurde nach den Aufnahmen die jeweils anwesende Erzieherin gefragt, ob sich das Zielkind anders verhalten habe als üblich. Wurde diese Frage von den Erzieherinnen bejaht, wurden die Aufnahmen aus den Analysen ausgeschlossen.

Das Zielkind wurde von der Filmerin während 30 Minuten durchgängig videographiert, unabhängig davon, ob sich das Kind in Kontakt mit anderen Kin- 
dern oder der Erzieherin befand oder für sich alleine spielte. Die Aufnahmen wurden lediglich unterbrochen, wenn das Zielkind ausgesprochen müde oder hungrig war oder pflegerische Aktivitäten von Seiten der Erzieherin notwendig waren. Befand sich das Zielkind am Ende der Aufnahmeperiode gerade in einer Interaktion, wurde diese noch vollständig gefilmt. Die Erzieherinnen wurden angewiesen, zurückhaltend zu agieren und sich nur so viel wie unbedingt nötig in das Geschehen einzumischen. Nahm ein Zielkind von sich aus mit der Erzieherin Kontakt auf, durfte diese darauf eingehen. Sie sollte jedoch vermeiden, mit den Zielkindern von ihr strukturierte Aktivitäten durchzuführen. Ebenso zurükkhaltend verhielten sich auch die Filmerinnen. Sie wurden angewiesen, nicht von sich aus mit den Zielkindern in Kontakt zu treten. Kamen die Zielkinder auf die Filmerinnen zu, verhielten sich diese freundlich-passiv.

Basisanalysen und Kodierung des prosozialen Verhaltens Von den möglichen 252 Videoaufnahmen (9 Zeitpunkte x 28 Zielkinder) standen 239 Filme $^{3}$ von je 30 Minuten für die Analysen zur Verfügung.

Für die Auswertung der Videodaten wurde die Software Observer Video Pro von Noldus verwendet und den Bedürfnissen entsprechend mit einem standardisierten Kategoriensystem konfiguriert. Inhaltlich lag den Kategorien das Konzept der geteilten Bedeutungen zugrunde, wie es von Brenner und Mueller (1982) entwickelt und von Viernickel (2000) weiter ausgebaut wurde. Viernikkel definiert die Bedeutung einer Interaktionseinheit als «das vom Kind in einer Interaktionssituation wahrgenommene Thema, nach dem es seine Handlungen ausrichtet» (Viernickel, 2000, S. 97). Die subjektive Bedeutung, die ein Interaktionsgeschehen für ein Kind hat, kann vom Beobachter aufgrund des Verhaltens des Kindes und seiner Partner gefolgert werden. Die Themenliste von Viernickel wurde leicht modifiziert, damit das Verhalten der Kinder zu den frühen Aufnahmezeitpunkten adäquat erfasst werden konnte. Für jede Verhaltenseinheit wurden Zeitpunkt und Dauer des Auftretens sowie bei Interaktionen die involvierte(n) Partner und das Teilen/Nichtteilen der Bedeutung festgehalten. Innerhalb jeder thematischen Einheit wurden ausserdem konkrete Verhaltensweisen erfasst (z. B. Objekt anbieten, umarmen, schlagen, anlächeln etc.).

Diese Grundauswertung diente als Basis für die Identifizierung und Auswertung von 998 Szenen, die prosoziales Verhalten aufwiesen oder Gelegenheit dazu boten. $\mathrm{Zu}$ letzteren wurden Konflikte oder Situationen, in denen eine anwesende Person weinte, gezählt.

Wenn das Zielkind prosoziales Verhalten zeigte, wurde dieses nach folgenden Formen getrennt weiter analysiert:

- Anbieten von Objekten: Überlassen eines Objekts zur ständigen oder temporären Benutzung

- Zuneigung zeigen: Einer anderen Person seine Zuneigung bekunden, ohne dass sich diese in einer Notlage befindet 
- Assistieren: Unterstützen in einer nicht emotionalen Notlage

- Trösten: Unterstützen in einer emotionalen Notlage, die durch den Gefühlsausdruck der anderen Person angezeigt wird

- Wiedergutmachung: Das Kind versucht ein Leid, das es einer anderen Person zugefügt hat, wieder gutzumachen.

- prosoziales Verhalten gegenüber Objekten: Empfänger des prosozialen Verhaltens ist ein unbelebter Gegenstand, z. B. eine Puppe.

Neben der Form des prosozialen Verhaltens wurde festgehalten, an wen (Kind/Erwachsene, Geschlecht) die Zielkinder das Verhalten richteten, ob das Verhalten spontan, d.h. ohne Aufforderung einer anderen Person, ausgeführt wurde, ob ein Auslöser beobachtbar war und wie die Empfänger reagierten.

Zur Berechnung der Reliabilität wurde ein Teil des Videomaterials von einer zweiten wissenschaftlichen Mitarbeiterin doppelkodiert. Diese war an der Hauptstudie beteiligt und in der Videobeobachtung geschult, aber mit den spezifischen Fragestellungen der vorliegenden Studie nicht vertraut. Grundlage für die Reliabilitätsberechnungen waren 400 Szenen, die zufällig ausgewählt wurden. Aufgrund der Anlage der Studie konnte Cohens Kappa nicht berechnet werden, approximativ werden deshalb die prozentuale Übereinstimmungen zwischen den Kodiererinnen angegeben: Form des prosozialen Verhaltens: 79.00\%, prosozial gehandelt ja/nein: 94.75\%, Vorhandensein und Art des Auslösers: 81.75\% sowie Reaktion des Empfängers: 78.50\%. Für die statistischen Analysen wurden die Kodierungen der Erstautorin verwendet.

\section{Resultate}

Die statistische Analyse der Daten erfolgte mittels SPSS 16.0. Den Ausgangspunkt für die Beantwortung der eingangs aufgeworfenen Fragen bilden die Häufigkeiten der verschiedenen prosozialen Formen, gefolgt von der Erstmanifestation, dem Entwicklungsverlauf, dem möglichen Einflussfaktor Geschlecht und der Interkorrelation der untersuchten Formen prosozialen Verhaltens.

Häufigkeiten

Die Häufigkeit wurde für jede prosoziale Form getrennt berechnet und über die neun Messzeitpunkte aufsummiert (Abbildung 1). 


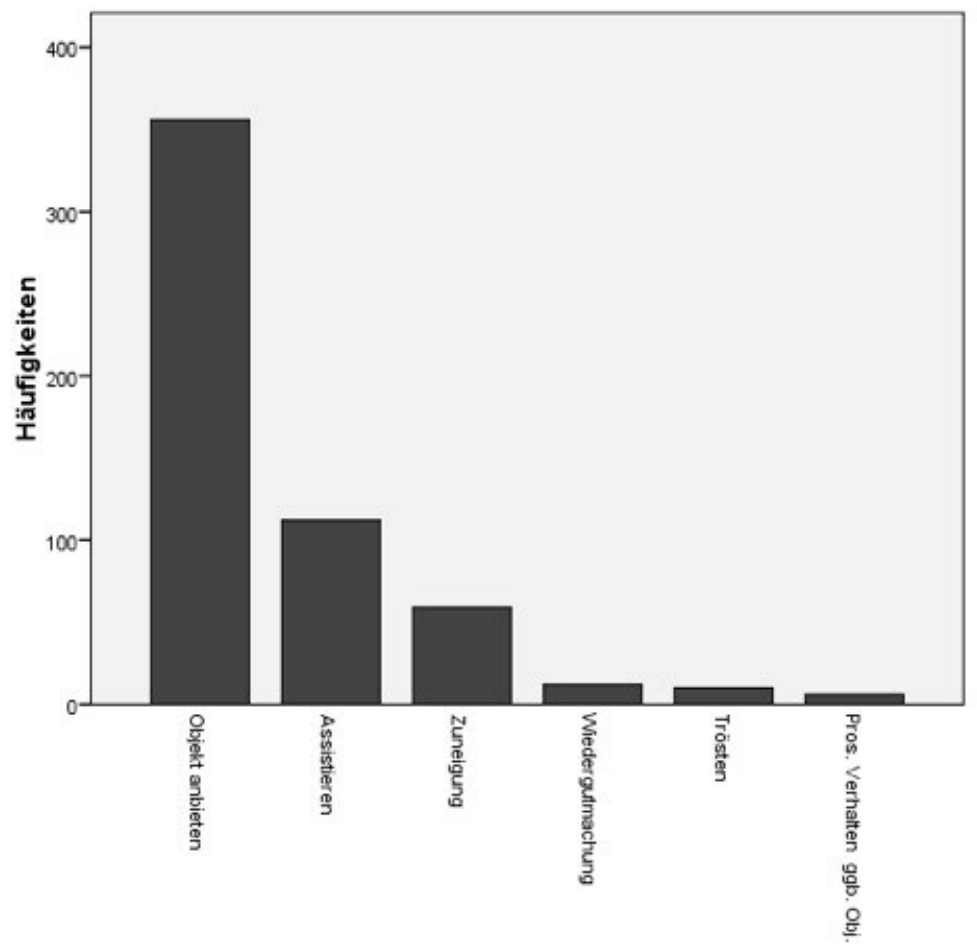

Abbildung 1: Häufigkeit der verschiedenen prosozialen Formen

Das Anbieten von Objekten war die mit Abstand häufigste Form von prosozialem Verhalten: Sie war über alle Messzeitpunkte hinweg insgesamt $356 \mathrm{Mal}$ beobachtbar. Dies entspricht einem prozentualen Anteil von 64\%, das heisst beinahe zwei Dritteln der analysierten Verhaltensweisen. Das Assistieren wurde von den Zielkindern $112 \mathrm{Mal}$ (20\%) gezeigt. Damit ist es nach dem Anbieten von Objekten die zweithäufigste Form, gefolgt von der Zuneigung, die bei den Zielkindern $59 \mathrm{Mal}(11 \%)$ beobachtet werden konnte. Nur selten kamen die übrigen Formen von prosozialem Verhalten vor: Trösten konnte $10 \mathrm{Mal}(1.8 \%)$ beobachtet werden, Wiedergutmachung $12 \mathrm{Mal}(2.2 \%)$ und prosoziales Verhalten gegenüber Objekten $6 \mathrm{Mal}(1 \%)$.

Offensichtlich sind beim Trösten und der Wiedergutmachung hemmende Faktoren wirksam. Dies wird verdeutlicht durch die Tatsache, dass sich viele Gelegenheiten zum Trösten und zur Wiedergutmachung ergeben hatten: Insgesamt weinten 139 Mal Peers in Hörweite des Zielkindes, jedoch nur in 10 Fällen griff das Zielkind tröstend ein, was einem Anteil von 7.2\% entspricht. Konflikte konnten ebenfalls häufig beobachtet werden, nämlich 326 Mal. Obwohl diese Situationen viele Gelegenheiten zur Wiedergutmachung boten, zeigten die Ziel- 
kinder dieses Verhalten lediglich $12 \mathrm{Mal}$, was einem Anteil von nur 3.7\% entspricht.

Das Anbieten von Objekten wurde als einzige Form von allen Zielkindern mindestens ein Mal gezeigt. Auch Assistieren und Zuneigung wurden von einer grossen Zahl der Kinder ausgeführt: 26 von 28 Kinder assistierten mindestens ein Mal im Verlauf der neun Messzeitpunkte, was einem prozentualen Anteil von $93 \%$ entspricht. $82 \%$ der Zielkinder, also 23, zeigten mindestens ein Mal einer anderen Person ihre Zuneigung. Auch hier wird ersichtlich, dass die genannten Verhaltensweisen nicht nur häufiger vorkommen als andere, sondern auch ein grosser Teil der Kinder diese Formen von prosozialem Verhalten zeigt. Obwohl die Wiedergutmachung eine sehr geringe Häufigkeit aufweist, nämlich 12, verteilt sich diese Verhaltensweise auf 10 Zielkinder (36\%). Dagegen versuchten lediglich 5 Kinder (18\%) der Stichprobe mindestens ein Mal, andere zu trösten. Noch geringer fällt diese Zahl beim prosozialen Verhalten gegenüber Objekten aus: 4 Kinder resp. 14\% zeigten diese Verhaltensweise.

\section{Erstmanifestation}

Die Resultate zur Erstmanifestation basieren auf der deskriptiven Darstellung der Häufigkeit der einzelnen prosozialen Formen pro Messzeitpunkt.

Das Anbieten von Objekten liess sich als früheste Form prosozialen Verhaltens bei zwei Zielkindern bereits mit 8 Monaten beobachten. Als nächstes waren das Zeigen von Zuneigung im Alter von 10 Monaten und das Assistieren mit 12 Monaten beobachtbar. Die Kategorie Wiedergutmachung zeigte sich erstmals mit 14 Monaten. Am spätesten manifestierten sich das Trösten und prosoziales Verhalten gegenüber Objekten mit 16 Monaten. Bereits mit 16 Monaten liessen sich bei den Zielkindern also alle sechs untersuchten prosozialen Verhaltensweisen beobachten.

Diese Resultate zeigen, dass sich das Erstmanifestationsalter je nach Form des prosozialen Verhaltens beträchtlich unterscheidet: Während das Anbieten von Objekten bei einigen Zielkindern mit 8 Monaten bereits im Verhaltensrepertoire vorhanden war, traten Trösten und prosoziales Verhalten gegenüber Objekten erst 8 Monate später erstmals auf. Zudem fällt auf, dass zu jedem Messzeitpunkt mindestens eine neue prosoziale Verhaltensform beobachtet werden konnte. Der Zeitraum zwischen 8 und 16 Monaten scheint also eine wichtige Entwicklungsphase für prosoziales Verhalten und ihm zugrunde liegende Entwicklungsschritte zu sein.

Verlauf

In Abbildung 2 ist die summierte Häufigkeit der beobachteten prosozialen Formen im Verlauf dargestellt. 


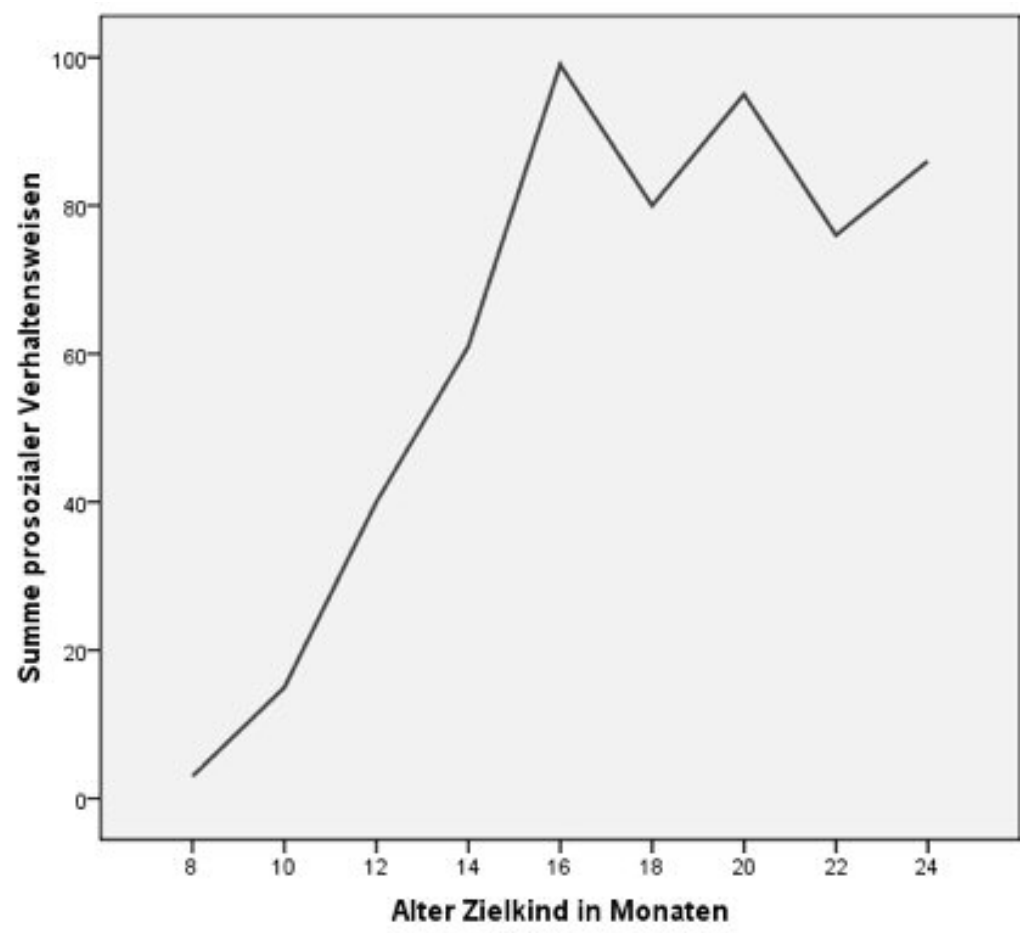

Abbildung 2: Verlauf der aufsummierten Häufigkeiten prosozialer Verhaltensweisen von 8 - 24 Monaten

Die Häufigkeit des prosozialen Verhaltens vom ersten Messzeitpunkt bis zum Alter von 16 Monaten nimmt stark zu. Dies ist darauf zurückzuführen, dass in diesem Zeitabschnitt einerseits neue Formen auftauchen und andererseits die Häufigkeit der einzelnen Formen zunimmt. Mit 16 Monaten scheint jedoch ein Plateau erreicht zu sein: Ab diesem Zeitpunkt ist keine eindeutige Zunahme mehr zu verzeichnen, allerdings auch keine klare Abnahme.

Um den Anstieg bei den ersten Messzeitpunkten und die nachfolgende Abflachung ab dem Alter von 16 Monaten statistisch zu überprüfen, wurden lineare gemischte Modelle mit einer autoregressiven, heterogenen Kovarianzstruktur mit dem Messzeitpunkt als wiederholter Faktor, in varianzanalytischen Designs als within-subject Faktor bezeichnet, gerechnet. ${ }^{4}$

Der Faktor Messzeitpunkt hatte einen signifikanten Effekt auf das prosoziale Verhalten der Zielkinder $(N=28 ; F(8,37)=18.02 ; p<.01$, ES $=0.5)$. Die paarweisen Einzelvergleiche ergaben, dass ein signifikanter Anstieg des prosozialen Verhaltens bis und mit dem fünften Messzeitpunkt zu verzeichnen ist. Die Mittelwerte der jeweiligen Messzeitpunkte unterscheiden sich signifikant von den jeweils vorhergehenden. Ab dem fünften Messzeitpunkt sind keine signifi- 
kanten Zunahmen mehr nachweisbar. Die Abnahmen zu den Zeitpunkten T6 und T8 sind ebenfalls nicht signifikant. Die Resultate bestätigen also den Eindruck des grafischen Verlaufs, dass das prosoziale Verhalten bis und mit dem fünften Messzeitpunkt (16 Monate) zunimmt, danach aber auf dem gleichen Niveau verbleibt.

In einem nächsten Schritt wurden für jede prosoziale Form gemischte Modelle gerechnet, deren Resultate in Tabelle 1 dargestellt sind.

Tabelle 1: Gemischte Modelle: Haupteffekt Zeitpunkt, differenziert nach der prosozialen Form

\begin{tabular}{|lll|}
\hline & $\mathrm{df}$ & $\mathrm{F}$ \\
\hline Objekt anbieten & 8,36 & $5.99^{* *}$ \\
Assistieren & 8,159 & $5.11^{* *}$ \\
Trösten & 8,151 & $2.29^{*}$ \\
Zuneigung & 8,151 & 1.33 \\
Wiedergutmachung & 8,156 & 1.50 \\
Empfänger: Objekt & 8,175 & 1.54 \\
\hline
\end{tabular}

Anmerkung: $\mathrm{N}=28 ;{ }^{* *} \mathrm{p}<.01 ;{ }^{*} \mathrm{p}<.05$

Für das prosoziale Verhalten gegenüber Objekten und die Wiedergutmachung konnten keine signifikanten Zeiteffekte nachgewiesen werden. Diese zwei Formen bewegen sich ab der Erstmanifestation vielmehr konstant auf einem niedrigen Niveau. Bei drei Formen resultierten signifikante Haupteffekte des Messzeitpunkts, nämlich für Objekt anbieten, Assistieren sowie Trösten. Hier wurden die einzelnen Zeitpunkte auch paarweise verglichen.

Für die Kategorie Objekt anbieten erwies sich die Zunahme lediglich bis zum dritten Zeitpunkt als signifikant. Somit steigt die Häufigkeit des Anbietens von Objekten bis 12 Monate an und verbleibt danach auf hohem Niveau.

Das Assistieren nimmt kontinuierlich leicht zu, mit signifikanten Anstiegen beim fünften und beim neunten Messzeitpunkt, also im Alter von 16 und 24 Monaten.

Das Trösten wurde von den Zielkindern erstmals mit 16 Monaten gezeigt, also beim fünften Messzeitpunkt. Eine zweite signifikante Zunahme ist beim siebten Messzeitpunkt, im Alter von 20 Monaten, zu verzeichnen. Einschränkend ist hier anzumerken, dass die Häufigkeit dieser Kategorie insgesamt sehr gering ist.

Die Resultate zeigen, dass sich die Verläufe der verschiedenen Formen deutlich voneinander unterscheiden: Einzig beim Assistieren ist eine beinahe konstante Zunahme über die Zeit zu verzeichnen, das Anbieten von Objekten nimmt dagegen nur bis 12 Monate zu. 


\section{Geschlechtsunterschiede}

Um Geschlechtsunterschiede zu überprüfen, wurden lineare gemischte Modelle mit einer autoregressiven, heterogenen Kovarianzstruktur gerechnet. Dabei zeigte sich ein signifikanter Haupteffekt des Geschlechts $(N=28, F(1,53)=$ $8.43, p<.01, \mathrm{~d}=0.2)$ : Mädchen handelten insgesamt häufiger prosozial als Jungen. Weitere Analysen ergaben, dass dieses Ergebnis auf nicht spontan auftretendes prosoziales Verhalten zurückzuführen ist. Kein Geschlechtsunterschied zeigte sich nämlich bezüglich der Untergruppe spontan auftretender prosozialer Verhaltensweisen.

\section{Interkorrelation}

Um die Frage zu beantworten, wie die einzelnen Formen von prosozialem Verhalten miteinander zusammenhängen, wurden bivariate Korrelationen über alle Messzeitpunkte hinweg gerechnet. Die Interkorrelationen sind in Tabelle 2 dargestellt.

Tabelle 2: Interkorrelationen der prosozialen Verhaltensweisen

\begin{tabular}{|lcccccc|}
\hline & $\begin{array}{c}\text { Objekt } \\
\text { anbieten }\end{array}$ & Assistieren & Trösten & $\begin{array}{c}\text { Wiedergut- } \\
\text { machung }\end{array}$ & Zuneigung & $\begin{array}{c}\text { Empfänger: } \\
\text { Objekt }\end{array}$ \\
\hline Objekt anbieten & $-/-$ & .097 & .060 & .035 & .066 & -.010 \\
Assistieren & & $-/-$ & .044 & .046 & .106 & -.021 \\
Trösten & & & $-/-$ & .111 & .063 & $.169^{* *}$ \\
Wiedergutmachung & & & & $-/-$ & .026 & .068 \\
Zuneigung & & & & & $-/-$ & $.247^{* *}$ \\
Empfänger: Objekt & & & & & & $-/-$ \\
\hline
\end{tabular}

Anmerkung: $\mathrm{N}=28 ;{ }^{* *} \mathrm{p}<.01$ (2-seitig)

Die Korrelationen zwischen den einzelnen prosozialen Formen sind mit zwei Ausnahmen niedrig. Sie reichen von .026 zwischen Zuneigung und Wiedergutmachung bis 247 zwischen Empfänger: Objekt und Zuneigung. Lediglich zwei Korrelationen fallen signifikant aus, nämlich zwischen prosozialem Verhalten gegenüber Objekten zum einen mit Zuneigung zeigen und zum andern mit Trösten. Die Korrelation zwischen scheinbar verwandten prosozialen Formen wie Wiedergutmachung und Trösten, die nach Hoffman (1975) auf der Empathie beruhen, liegt bei .111 und verfehlt knapp die Signifikanzgrenze. Die verschiedenen Formen von prosozialem Verhalten hängen also kaum zusammen. 


\section{Diskussion}

Die Resultate zeigen, dass schon sehr kleine Kinder zu prosozialem Verhalten fähig sind und bis zum Alter von zwei Jahren ein breites Repertoire an unterschiedlichen Formen aufgebaut haben.

Im Folgenden werden die Hauptresultate der Studie zusammengefasst und kurz diskutiert:

Erstmanifestation: Erste prosoziale Verhaltenswiesen waren bei zwei Zielkindern bereits mit acht Monaten Bestandteil ihres Verhaltensrepertoires, im Alter von 16 Monaten konnten bereits alle von uns untersuchten Formen des prosozialen Verhaltens beobachtet werden. Zwischen den verschiedenen Formen zeigten sich jedoch beträchtliche Unterschiede in Erstmanifestationsalter, Häufigkeit und Verlauf. Dies deutet darauf hin, dass es sich um Verhaltensweisen mit unterschiedlichen sozio-kognitiven, emotionalen sowie selbstregulatorischen Anforderungen und wahrscheinlich auch unterschiedlichen zugrunde liegenden Motiven handelt. Diese Schlussfolgerung wird durch die niedrigen Interkorrelationen zwischen den einzelnen Formen gestützt.

Dass das Anbieten von Objekten so früh gezeigt wird, ist kongruent mit Modell sekundärer Kreisreaktionen (Piaget, 1981): Kennzeichnend für diese Phase ist, dass das Kind Mittel und Zweck voneinander unterscheiden und bestimmte Handlungen, wie zum Beispiel das Anbieten von Objekten, unter anderem als Mittel zur Kontaktaufnahme einsetzen kann. Ähnliches gilt für das Zeigen von Zuneigung, für das dieselben kognitiven Voraussetzungen notwendig sind. Dass diese Verhaltensweise zwei Monate später als das Objekte anbieten auftritt, könnte damit zusammenhängen, dass sie ein aktiveres Vorgehen des Akteurs erfordert.

An weitere sozio-kognitive und motorische Voraussetzungen gebunden ist das Assistieren: Die Möglichkeit zur Assistenz wird durch Signale der Situation angezeigt. Insbesondere muss das intendierte, aber unerreichte Ziel der anderen Person erschliessbar sein. Voraussetzung hierfür ist nach Karniol (1982), dass das Kind entsprechende Wissensstrukturen in Form von allgemeinen Schemata und Skripts aufgebaut haben muss. Verschiedene Autoren (Gergely \& Csibra, 2003; Tomasello \& Rakoczy, 2003) konnten zeigen, dass Kinder mit 12 Monaten in der Lage sind, Absichten und intendierte Ziele anderer Personen zu erschliessen. Diese Angaben stimmen mit dem in der vorliegenden Studie gefundenen Erstmanifestationsalter überein. Da das Assistieren vom Kind meist eine räumliche Annäherung zu der Person oder der Situation erfordert, ist die Fähigkeit zur Lokomotion eine weitere wichtige Voraussetzung für die Ausübung dieser Form von prosozialem Verhalten.

Das Trösten und die Wiedergutmachung konnte erstmals mit 16 bzw. 14 Monaten beobachtet werden. Dieser Befund stimmt mit den Resultaten aus den Längsschnittstudien von Zahn-Waxler, Radke-Yarrow und Chapman (1992) 
überein: Die Kinder fingen in diesen Untersuchungen zwischen 13 und 15 Monaten an, erstmals prosozial auf das Leid anderer zu reagieren. Im Unterschied zum Assistieren wird beim Trösten und der Wiedergutmachung die Hilfsbedürftigkeit durch den mimischen Ausdruck der anderen Person angezeigt. Das Kind muss also in der Lage sein, vom mimischen Ausdruck auf den inneren Zustand einer Person zu schliessen. Weiter ist die Situation im Unterschied zum Assistieren von negativen Emotionen des potentiellen Empfängers geprägt. Nach Eisenberg et al. (1996) sind in emotional negativ gefärbten Situationen zusätzlich Fähigkeiten zur Selbstregulation erforderlich: Die eigenen emotionalen Reaktionen, die bei der Beobachtung einer leidenden Person entstehen, müssen innerhalb eines tolerierbaren Rahmens gehalten werden: Wird die eigene Erregung zu gross, so wird die stellvertretende induzierte Emotion als zu aversiv erlebt, was personal stress erzeugt. Dies führt dazu, dass das Kind auf sich selbst fokussiert bleibt und damit beschäftigt ist, die eigene Überregung zu bewältigen (Eisenberg et al., 1996).

Diese Resultate sind sowohl mit dem von Hoffman (1982) als auch von Bischof-Köhler (1989) postulierten Entwicklungsverlauf der Empathie vereinbar. Eine erste Differenzierung zwischen Gefühlsansteckung und Einfühlungsvermögen scheint sich kurz nach dem ersten Geburtstag herauszubilden. Dies kann als Voraussetzung für prosoziale Reaktionen wie Trösten oder Wiedergutmachung gelten. Die Untersuchung von Vaish et al. (2009) zeigt ausserdem, dass Mitgefühl mit einer Person bereits bei unter Zweijährigen die Wahrscheinlichkeit erhöht, ihr in einer folgenden Situation zu helfen. Mit der Entwicklung eines Selbstkonzeptes und der damit verbundenen Fähigkeit zur Selbstobjektivierung, wie Bischof-Köhler sie postuliert, erweitern sich die Möglichkeiten komplexere soziale Situationen zu erfassen und Gefühle beim andern zu erschliessen, was zu vielfältigeren Handlungsoptionen führt.

Als letzte der untersuchten prosozialen Verhaltensweisen trat prosoziales Verhalten gegenüber Objekten auf. Es dürfte sich dabei um eine Form des Symbolspiels handeln, die sich Mitte des zweiten Lebensjahres, also mit dem frühen einfachen Symbolspiel (Belsky \& Most, 1981) zeigt.

Im weiteren Verlauf bis zwei Jahre zeigen sich Unterschiede zwischen den verschiedenen Formen von prosozialem Verhalten. Das Anbieten von Objekten konnte bereits beim ersten Messzeitpunkt beobachtet werden. In der Folge steigt die Häufigkeit bis zum dritten Messzeitpunkt an. Danach ist jedoch keine $\mathrm{Zu}-$ nahme mehr festzustellen, vielmehr verbleibt das Anbieten von Objekten auf einem hohen Niveau. Offensichtlich sind die für die Ausführung dieses Verhaltens notwendigen Voraussetzungen schon früh in der Entwicklung vorhanden. Diese Resultate stimmen mit der Beobachtung von Stanjek (1978) überein. In seinen Untersuchungen nahm die Häufigkeit des Anbietens von Objekten bis zum Alter von zwei Jahren zu und sank danach. Bis zum zweiten Lebensjahr hat das Anbieten von Objekten nach Stanjek (1978) die Funktion, mit anderen Personen in Kontakt zu treten. Mit den zunehmenden verbalen Fähigkeiten der Zielkin- 
der und einem immer breiter werdenden Repertoire an zusätzlichen Möglichkeiten, mit anderen in Kontakt zu treten und Interaktionen aufrechtzuerhalten, wird das Anbieten von Objekten nach dem zweiten Lebensjahr von anderen Strategien abgelöst.

Das Assistieren dagegen nimmt bis zum zweiten Lebensjahr zu. Offenbar erwerben immer mehr Kinder der Stichprobe die notwendigen Voraussetzungen. Ausserdem ist davon auszugehen, dass sich die anfangs rudimentären Skripts und Schemata zunehmend mit Inhalten füllen. Dies führt dazu, dass die Kinder immer mehr und subtilere Signale der Hilfsbedürftigkeit erkennen und sich das Wissen darüber, wie prosozial gehandelt werden kann, ständig erweitert. Beim Trösten konnten ebenfalls signifikante Alterseffekte festgestellt werden: Die Häufigkeit ist beim fünften und siebten Messzeitpunkt höher als bei den übrigen. Allerdings kommt das Trösten insgesamt lediglich $12 \mathrm{Mal}$ vor, weshalb diese Resultate auf zufällige Schwankungen zurückzuführen sein könnten. Bei den übrigen prosozialen Verhaltensweisen konnte kein eindeutiger Verlauf festgestellt werden. Im Fall der Wiedergutmachung und dem prosozialen Verhalten gegenüber Objekten könnte dies damit zusammenhängen, dass sie selten vorkommen und somit keine zuverlässigen Aussagen über Verläufe möglich sind. Beim Zeigen von Zuneigung hat das Alter keinen Einfluss auf den Verlauf. Dies deckt sich mit den Aussagen von Eisenberg und Mussen (1989), wonach bei solchen Verhaltensweisen der Einfluss der Persönlichkeit des Zielkindes stärker ist als derjenige des Alters.

Die untersuchten prosozialen Verhaltensweisen weisen je nach Form unterschiedliche Häufigkeiten auf. Das Anbieten von Objekten kommt sehr häufig vor und wurde von allen Zielkindern mindestens ein Mal gezeigt. Eine Verhaltensweise, die wahrscheinlich einem ähnlichen Zweck dient, aber seltener vorkommt, ist das Zeigen von Zuneigung. Es erfordert ein aktiveres Vorgehen und wird vermutlich eher von Kindern gezeigt, die einen aktiven sozialen Stil aufweisen.

Als zweithäufigste prosoziale Verhaltensweise erweist sich das Assistieren. Dies deckt sich mit der zitierten Studie (Rheingold, 1982), bei denen die Kinder bereitwillig bei der Erledigung von diversen Aufgaben halfen. Auch in der vorliegenden Studie halfen die Kinder zügig bei der Erledigung von verschiedenen Aufgaben. Aus der Tatsache, dass die Kinder häufig spontan halfen, kann geschlossen werden, dass sie das intendierte Ziel verstanden hatten (Warneken \& Tomasello, 2007).

Trösten und die Wiedergutmachung sind selten vorkommende Verhaltensweisen. Insgesamt werden sie über alle Zielkinder und Zeitpunkte nur 12 resp. $10 \mathrm{Mal}$ gezeigt. An Gelegenheiten zur Wiedergutmachung und zum Trösten fehlte es allerdings nicht, die Zielkinder waren einerseits in Konflikte involviert und andererseits weinten häufig andere Kinder in ihrer unmittelbaren Nähe. Dies deutet darauf hin, dass hier stark hemmende Faktoren wirksam sein müssen. Zur theoretischen Einordnung bieten sich mehrfache Möglichkeiten: Nach Eisenberg und Fabes (1998) kann das Zielkind in emotionalen Situationen nur 
dann prosozial handeln, wenn die Notsituation erkannt wird und die eigene Erregung in bestimmten Grenzen gehalten werden kann. Wird sie dagegen zu gross, bleibt das Kind auf sich selbst fokussiert und ist damit beschäftigt, der eigenen Erregung Herr zu werden. In unserer Stichprobe zeigten die Zielkinder jedoch selten Anzeichen einer hohen Erregung. Bischof-Köhler (1989) dagegen glaubt, dass die Wahrscheinlichkeit einer prosozialen Intervention sinkt, wenn die Kinder in eine interessante Tätigkeit, wie z. B. ein Spiel, vertieft sind. Caplan (1993) vermutet, dass Kinder häufig keine Gelegenheit bekommen, prosozial zu reagieren, da Erzieherinnen normalerweise sehr schnell tröstend eingreifen, wenn ein Kind weint. Dadurch vermitteln sie den Kindern, dass es die Aufgabe von Erwachsenen ist, weinende Kinder zu trösten. Die Annahme von Caplan wird gestützt durch die Beobachtung, dass die Erzieherinnen in unserer Stichprobe tatsächlich meist schnell auf ein weinendes Kind reagierten.

Mädchen handeln signifikant häufiger prosozial als die Jungen. In Übereinstimmung mit den Resultaten der hier vorgestellten Studie schliessen Eisenberg und Fabes (1998) in ihrer Metaanalyse, dass gefundene Geschlechtsunterschiede zugunsten der Mädchen ausfielen. Nach Eisenberg und Mussen (1989) werden bei Jungen und Mädchen unterschiedliche Erziehungstechniken angewendet, die dazu führen, dass prosoziales Verhalten von Mädchen gefördert, bei Jungen hingegen gehemmt wird. Des Weiteren werden Mädchen für prosoziales Verhalten häufiger positiv verstärkt als Jungen. In der vorliegenden Studie konnte jedoch nicht gezeigt werden, dass die Mädchen von den Empfängern des Verhaltens oder von Drittpersonen häufiger positiv verstärkt wurden als die Jungen. Allerdings wurden lediglich bestimmte, offen gezeigte Reaktionen auf das prosoziale Verhalten der Zielkinder erfasst und andere, subtile Formen der Verstärkung ausgeklammert.

Es könnte ausserdem sein, dass Mädchen aufgrund geschlechtsstereotyper Vorstellungen häufiger zu prosozialem Verhalten aufgefordert werden als Jungen. Diese Erklärung ist kongruent mit dem Ergebnis der eigenen Studie, die für spontan auftretendes prosoziales Verhalten keine Geschlechtsunterschiede gezeigt hat. Die gefundenen Geschlechtsunterschiede könnten also darauf zurükkzuführen sein, dass Mädchen einerseits häufiger zu prosozialem Verhalten aufgefordert werden und andererseits angepasster und folgsamer als die Jungen der Aufforderung nachkommen.

Die naheliegende Vermutung, dass die Mädchen einen generellen Entwikklungsvorsprung gegenüber den Jungen aufweisen könnten, der sich auch beim prosozialen Verhalten zeigt, kann durch die vorliegende Studie nicht gestützt werden: In einer allgemeinen Entwicklungsuntersuchung (Griffith Entwikklungsskalen; Brandt \& Sticker, 2001), die im Alter von 16 Monaten durchgeführt wurde, unterschieden sich Jungen und Mädchen nicht signifikant voneinander.

Die Studie konnte einen Beitrag zur Schliessung einiger Forschungslücken leisten und die Entwicklung des prosozialen Verhaltens in den ersten zwei Le- 
bensjahren längsschnittlich dokumentieren. Es konnte aufgezeigt werden, dass schon sehr kleine Kinder zu prosozialem Verhalten fähig sind und bis zum Alter von zwei Jahren ein breites Repertoire an unterschiedlichen Formen aufgebaut haben. Zwischen den verschiedenen Formen zeigen sich Unterschiede in Erstmanifestationsalter, Häufigkeit und Verlauf. Dies deutet darauf hin, dass es sich hierbei um Verhaltensweisen mit unterschiedlichen sozio-kognitiven, emotionalen sowie selbstregulatorischen Anforderungen und wahrscheinlich auch unterschiedlichen zugrunde liegenden Motiven handelt.

Abschliessend soll angemerkt werden, dass die Ergebnisse der vorliegenden Untersuchungen aus folgenden Gründen beschränkt generalisierbar sind:

- Die vorliegende Untersuchung ist nicht repräsentativ: Sie ist auf städtische Einrichtungen im Raum Zürich beschränkt und dabei auf die freiwillige Beteiligung der Kindertagesstätten und der Familien angewiesen.

- Die Zielkinder und die Kindertagesstätten unterschieden sich hinsichtlich wichtiger Merkmale wie bspw. Temperament bzw. Gruppenstrukturen erheblich voneinander. Was die Familien der Kinder betrifft waren Eltern mit einem hohen Ausbildungsniveau übervertreten. Diese Faktoren konnten in unserer Studie nicht ausbalanciert oder kontrolliert werden.

Um die in der vorgestellten Untersuchung gefundenen Resultate zu replizieren und zu generalisieren, bedarf es weiterer Studien mit repräsentativen Stichproben, die vertiefend spezifische Fragestellungen bearbeiten.

\section{Anmerkungen}

1 Egozentrimus bedeutet in diesem Zusammenhang, dass die Kinder lediglich die eigene Perspektive wahrnehmen können und kognitiv noch nicht in der Lage sind, sich in die Lage einer anderen Person zu versetzen

2 Die Studie wurde vom Schweizerischen Nationalfonds SNF (Nr. 11-63574-00) und von einer Stiftung finanziell unterstützt.

3 Insgesamt 13 Aufnahmen konnten für die Analyse nicht verwendet werden, da die Zielkinder gegenüber der Filmerin einmalig heftig fremdelten, krank oder abwesend waren.

4 Gegenüber Varianzanalysen mit Messwiederholung weisen gemischte Modelle für die vorliegende Untersuchung eine Reihe von Vorteilen auf. So gilt bei varianzanalytischen Designs, dass die Anzahl der Probanden «um einiges grösser» sein sollte als die Anzahl der abhängigen Variablen, die vorliegende Studie weist jedoch einen kleinen Stichprobenumfang von nur 28 Kindern auf. Im Gegensatz zu varianzanalytischen Designs werden bei den gemischten Modellen bei wiederholten Messungen die Werte eines Subjekts nicht in einer Zeile der Datendatei erwartet sondern in verschiedenen Zeilen, womit sich die Fallzahl erhöht. Dabei sind auch unvollständige Messwiederholungen erlaubt, die Anzahl der Beobachtungen darf also für die Subjekte variieren. 


\section{Literaturverzeichnis}

Belsky, J. \& Most, R. J. (1981). From exploration to play: A cross-sectional study of infant free play behavior. Developmental Psychology, 17, 630-639.

Bischof-Köhler, D. (1989). Spiegelbild und Empathie. Die Anfänge der sozialen Kognition. Bern: Hans-Huber.

Brandt, I. \& Sticker, E. J. (2001). GES Griffith-Entwicklungsskalen zur Beurteilung der Entwicklung in den ersten beiden Lebensjahren. Göttingen: Beltz Test GmbH.

Brenner, J. \& Mueller, E. (1982). Shared meaning in boy toddler's peer relation. Child Development, 53, 380-391.

Caplan, M. (1993). Inhibitory influences in development: The case of prosocial behavior. In D. F. Hay \& A. Angold (Ed.), Precursors and causes in development and psychopathology (pp. 169-198). Chichester: John Wiley \& Sons.

Eisenberg, N. \& Fabes, R. A. (1998). Prosocial development. In N. Eisenberg (Ed.), Social, emotional, and personality development (Vol. 3, pp. 701-778). New York: John Wiley \& Sons.

Eisenberg, N., Fabes, R. A., Murphy, B., Karbon, M., Smith, M. \& Maszk, P. (1996). The relations of children's dispositional empathy-related responding to their emotionality, regulation, and social functioning. Developmental Psychology, 32, 195-209.

Eisenberg, N. \& Mussen, P. H. (1989). The roots of prosocial behavior in children. Cambridge: University Press.

Eisenberg, N. (1986). Altruistic Emotion, Cognition, and Behavior. Hillsdale: Lawrence. Erlbaum Associates Publishers

Freud, S. (2009). Abriss der Psychoanalyse: Einführende Darstellungen. Frankfurt am Main: Fischer Taschenbuch Verlag.

Gergely, G. \& Csibra, G. (2003). Teleological reasoning in infancy: the naïve theory of rational action. Trends in Cognitive Sciences, 7, 287-292.

Harris, P. L. (1992). Das Kind und die Gefühle. Wie sich das Verständnis für die anderen Menschen entwickelt (M. Wengeroth, Übers.). Bern: Huber. (Originalarbeit erschienen 1989)

Hoffman, M. L. (1975). Developmental synthesis of affect and cognition and its implications for altruistic motivation. Developmental Psychology, 11, 607-622.

Hoffman, M. L. (1982). Development of prosocial motivation: Empathy and guilt. In N. Eisenberg (Ed.), The development of prosocial behavior (pp. 281-313). New York: Academic Press.

Karniol, R. (1982). Settings, scripts, and self-schemata: a cognitive analysis of the development of prosocial behavior. In N. Eisenberg (Ed.), The development of prosocial behavior (pp. 251-278). New York: Academic Press.

Keller, M. (2005). Moralentwicklung und moralische Sozialisation. In D. Horster \& J. Oelkers (Hrsg.), Pädagogik und Ethik (S. 149-172). Wiesbaden: Verlag für Sozialwissenschaften.

Kochanska, G. (1984). Regulatory theory of personality and the development of prosocial behavior. In E. Staub, D. Bar-Tal, J. Karylowski \& R. Reykowski (Ed.), Development and maintenance of prosocial behavior. International perspectives on positive morality (pp. 155176). New York: Plenum Press.

Kohlberg, L. (1976). Moral stages and moralization: The cognitive-developmental approach. In T. Lickona (Ed.), Moral development and behavior: Theory, research, and social issues (pp. 31-53). New York: Holt, Rinehart and Winston.

Piaget, J. (1981). Intelligence and affectivity: Their relation during child development. Palo Alto: Annual Reviews.

Rheingold, H. L. (1982). Little children's participation in the work of adults, a nascent prosocial behavior. Child Development, 53, 114-125.

Simoni, H., Herren, J., Kappeler, S. \& Licht, B. (2008). Frühe soziale Kompetenz unter Kindern. In: T. Malti \& S. Perren (Hrsg.), Entwicklung und Förderung sozialer Kompetenzen in Kindheit und Adoleszenz (S. 15-34). Stuttgart: Kohlhammer. 
Stanjek, K. (1978). Das Überreichen von Gaben: Funktion und Entwicklung in den ersten Lebensjahren. Zeitschrift für Entwicklungspsychologie und Pädagogische Psychologie, 10, 103113.

Tomasello, M. \& Rakocsy, H. (2003). What makes human cognition unique? From individual to shared to collective intentionality. Mind \& Language, 18, 121-147.

Vaish, A., Carpenter, M. \& Tomasello, M. (2009). Sympathy through affective perspective taking and its relation to prosocial behaviour in toddlers. Developmental Psychology, 45, 535543.

Viernickel, S. (2000). Spiel, Streit, Gemeinsamkeiten. Einblicke in die soziale Kinderwelt der unter Zweijährigen. Landau: Verlag Empirische Pädagogik.

Warneken, F. \& Tomasello, M. (2007). Helping and cooperation at 14 months of age. Infancy, 11, 271-294.

Zahn-Waxler, C. \& Radke-Yarrow, M. (1982). The development of altruism: Alternative research strategies. In N. Eisenberg (Ed.), The development of prosocial behavior (pp. 109137). New York: Academic Press.

Zahn-Waxler, C., Radke-Yarrow, M. \& Chapman, M. (1992). Development of concern for others. Developmental Psychology, 28, 126-136.

Schlagworte: Prosoziales Verhalten, frühe Kindheit, Längsschnittstudie

\section{Le développement des comportements protosociaux dans les deux premières années de la vie}

\section{Résumé}

Le développement des premiers comportements sociaux a été étudié de manière longitudinale chez 28 enfants de 9 à 25 mois à l'aide d'observations participantes en milieu naturel. Les comportements sociaux ont été définis comme des actions qui favorisent l'amélioration ou le rétablissement du bien être d'autres personnes ou d'un groupe. Six formes de comportements sociaux ont été étudiées: aider, consoler, montrer de la sympathie, offrir un objet, réparer et avoir des comportements sociaux avec des objets.

Les premières formes de comportements sociaux, par exemple offrir un objet sont déjà observées chez quelques enfants de 8 mois; à l'âge de 16 mois, elles peuvent être associées à d'autres comportements observés. La plupart des comportements se manifestent spontanément, sans sollicitation externe. Les formes de comportements prises séparément se distinguent considérablement s'il s'agit de premières manifestations ou de manifestations fréquentes ou considérées dans le déroulement temporel. Ceci signifie qu'aux différents comportements protosociaux sous-tendent des capacités cognitives différentes. Des différences liées au genre ont été mises en évidence: les filles manifestent plus fréquemment des comportements sociaux que les garçons.

Mots clés: Développement de l'enfant, comportements sociaux précoces, petite enfance, étude longitudinale 


\section{Lo sviluppo del comportamento pro sociale nei primi due anni di vita}

\section{Riassunto}

Uno studio longitudinale basato sullo sviluppo del comportamento prosociale è stato condotto su 28 bambini tra 9 e 25 mesi d'età nel loro ambiente naturale, servendosi dell'osservazione partecipante. Il comportamento prosociale è stato definito nel modo seguente: si tratta di azioni che aumentano o ristabiliscono il benessere di un'altra persona rispettivamente di un gruppo di persone oppure che creano vantaggi in loro favore.

Sono state analizzate sei forme di comportamento prosociale: prestare assistenza, consolare, mostrare affetto, offrire degli oggetti, ristabilire un buon rapporto come pure forme di comportamento prosociale verso gli oggetti. Singoli bambini manifestano forma prime forme di comportamento pro sociale, offrire degli oggetti, già all'età di 8 mesi. Prima dei 16 mesi sono state osservate tutte le forme esaminate. La gran parte dei comportamenti pro sociali si è manifestata in modo spontaneo e si è distinta per la prima apparizione, per la sequenza e loro per la successione cronologica. Ciò sta ad indicare come le diverse forme di comportamento pro sociale si fondi su differenti capacità socio-cognitive. Infine, le ragazze agiscono più frequentemente in maniera prosociale che ragazzi.

Parole chiave: Comportamente pro sociale, infanzia, studio longitudinale

\section{Development of prosocial behavior in the two first years of life}

\section{Abstract}

The development of pro-social behavior was researched in a longitudinal study with 28 children between 9 and 25 months old in their natural environment by a participant-observer. Pro-social behavior was thus defined as actions that enhance or restore the well being of another person or group or actions that favor another person or group. Six different types of pro-social behavior were studied: assisting, comforting, showing affection, offering objects, making up for/fixing, as well as pro-social behavior towards objects.

Primary types of pro-social behavior such as offering objects were already observed in children 8 months old. All of the studied types of pro-social behavior could be found in children up to 16 months old. Most of the pro-social behavior has been shown spontaneously. The different types of pro-social behavior vary considerably with regard to their first occurrence, their frequency and their development over time. This suggests that different pro-social behavior patterns are based on different socio-cognitive skills. Differences between boys and girls were observed: girls showed more pro-social behavior than boys.

Key words: Pro-social behavior, infancy, longitudinal study 